\title{
RADIOMICS BASED SINGLE AND MULTI-CLASS GLIOMA CLASSIFICATION USING SUPPORT VECTOR MACHINE VARIANTS
}

\author{
Seema P. D. ${ }^{1}$, Christy Bobby T. ${ }^{1}$ and Anandh K.R. ${ }^{2}$ \\ ${ }^{1}$ Ramaiah University of Applied Sciences, Bangalore, Karnataka, India. \\ ${ }^{2}$ University of Alabama at Birmingham, Birmingham, Alabama, United States.
}

Corresponding Author: Seema P. D.

Author's full Address: \#470-P, Peenya Industrial Area, Peenya 4th Phase, Bangalore - 560 058, Karnataka, India

Email: seemapd6@gmail.com

https://doi.org/10.34107/BiomedSciInstrum.57.04265

\begin{abstract}
The common type of primary brain tumor is glioma. The mortality rate of glioma patients is high due to delayed diagnosis, incorrect grading and treatment planning. Traditionally, gliomas were classified into Low Grade (grade-I and grade-II) and High Grade (grade-III and grade-IV). However, World Health Organization has insisted to classify the grades into grade-I(GI), grade II(G-II), grade III(G-III) and grade IV(G-IV) individually to aid the physicians in clinical decision-making. Although there are limited number of studies reported to differentiate individual grades, the classification accuracy was low. Consequently, in this work single-class (G-II vs. G-III, G-II vs. G-IV and G-III vs. G-IV) and multi-class (G-II vs. G-III+IV, G-III vs. G-II+IV and G-IV vs. G-II+III) analysis was performed using specific region of tumor and whole brain as Regions of Interest(ROI) by extracting radiomic features. The images for this study $(\mathrm{N}=75)$ were obtained from The Cancer Imaging Archive. Further, the statistically significant features were used in the classification of individual grades by implementing variants of Support Vector Machine (SVM) algorithm: SVM, Linear-SVM and Least-Squared SVM. Among these, LinearSVM resulted in the highest classification accuracy $(>80 \%)$ with average sensitivity, specificity and AUC values of $>70 \%$. The comparative analysis of whole brain versus tumor ROI showed that the latter yielded better classification accuracy.
\end{abstract}

Keywords: Glioma, Glioma Grading, Support Vector Machine

\section{INTRODUCTION}

Glioma is a type of primary brain tumor occurring in the glial cells of the brain accounting for $30 \%$ of all the primary brain tumors with a high mortality rate [1]. On the basis of presence of anaplastic features and degree of malignancy, World Health Organization has classified glioma to grade I(G-I), grade II(G-II), grade III(G-III) and grade IV(G-IV) of which G-I and II are Low Grade Glioma(LGG) and G-III and IV are High Grade Glioma(HGG) [2]. G-I is usually benign and can be removed by surgery alone [3]. There are less chances of it transitioning to G-II/III. G-II is benign but there are high chances of it progressing to G-III/ IV and it can be treated with a surgical resection. G-III is very sporadic, if identified on time, it can be controlled by surgical resection followed by radiation therapy. A combination of radiotherapy and chemotherapy after resection may help patients having G-IV glioma [3]. Since the treatment planning is based on the severity of the glioma, the classification into individual grade plays a very crucial role. Also, the approximate 5-year survival rates of G-II, G-III and G-IV are 50\%, 30\% and 5\% respectively [1].

Magnetic Resonance Imaging(MRI) is the most commonly applied imaging modality to diagnose glioma as it provides high resolution maps of anatomical structures with a high soft-tissue contrast, does not involve exposure to ionizing radiation and is less susceptible to artefacts [4]. T1-weighted gadolinium enhanced MRI is often preferred to image glioma. Gadolinium is the choice of contrast agent as it has the 